\title{
The Science of Situations and the Integration of Personality and Social Psychology
}

\author{
M. Brent Donnellan (Texas A \& M University)
}

Katherine S. Corker (Kenyon College)

Abstract: The Rauthmann, Sherman, and Funder (in press) target article provides a solid foundation for future research concerning the psychology of situations. Inspired by their work, we offer two extensions. First, we suggest that efforts be directed toward developing a working taxonomy of situational characteristics. Second, we suggest that experiments, particularly those rooted in classic experimental social psychology, may prove to be very useful for testing whether situational cues truly cause situational characteristics and ultimately behavior. In sum, we think their paper provides a template for integrating personality and social psychology.

Personality psychologists have developed and validated sophisticated taxonomies to classify the myriad ways people differ from one another. Although this topic still generates debate, current disagreements rarely overshadow the substantial progress made on this topic. More importantly, trait taxonomies have helped researchers systematically document how dispositions are associated with behavior and consequential life outcomes (Ozer \& Benet-Martínez, 2006; Roberts et al., 2007). This sanguine state of affairs does not seem to characterize the science of situations. Fortunately, this is changing as epitomized by the Rauthmann, Sherman, and Funder (in press; hereafter RSF) target article.

RSF's paper is rich, challenging, and ultimately successful in outlining a viable conceptual approach and set of principles for studying situations. They have provided readers with a compelling foundation for future research. Inspired by their work, we offer two suggestions.

\section{Focus on Situational Characteristics and let the Cues and Classes Follow}

RSF describe a tripartite model of situations involving cues, characteristics, and classes. We read characteristics as the psychologically relevant attributes of situations that are proximal causes of behavior. This facet of situations seems to be the most fruitful starting point for building a systematic understanding of the situation. A focus on situational characteristics may even help uncover robust evidence of interactions between situational factors and personality dispositions for predicting behavior, the Holy Grail for integrating personality and social psychology.

As it stands, we suspect it will be easier to identify specific cues once researchers have a firm grasp of the psychological dimensions that characterize a wide range of situations. Cues give rise to situational characteristics through information processing systems within individuals according to RSF's model. Accordingly, a well-articulated taxonomy of situational characteristics offers researchers a starting point for identifying cues. In fact, we suspect it will be difficult to identify cues without some understanding of their psychological effects. Further, 
the existence of situational classes can be addressed empirically, at least partially, with statistical tools designed to identify latent classes from characteristics (as noted by RSF).

Based on these considerations, we propose this mantra: Identify a useful and reasonably valid working taxonomy of situational characteristics and the cues and classes (if any) will follow. Fortunately, the toolkit of the typical personality psychologist (viz., expertise in psychological assessment) is well suited to this task. Moreover, RSF appear to have made good progress on this front.

\section{Experiments Have Something to Offer}

RSF's Figure 1 is a causal model, and we believe that experiments are a privileged method for bolstering causal inferences. However, we detected some reluctance by RSF to strongly endorse the use of experimental approaches for testing their ideas. For instance, RSF recommend that "research on person-situation transactions focus on natural situations" (p. 31). They seem to argue that because of the processes of selection, evocation, and manipulation (e.g., Buss, 1987), experimentally created situations lack utility for researchers. In contrast, we think there is a role for creative experimentation for advancing the science of the situation. The caveat is that the kinds of experiments we imagine are more firmly rooted in a working taxonomy of situational characteristics than has typically been the case.

As we see it, an important task for a science of situations is to identify cues that are (a) able to be manipulated by researchers; and (b) consistently related to situational characteristics (i.e., cues that generate reasonably similar psychological reactions in many people). People may disagree about the number of cues that fit these criteria, but we are optimistic. The Cyberball game is a good test case as it seems to reliably produce large effect sizes (meta-analytic $d=1.36 ; 95 \% \mathrm{CI}=$ [1.18, 1.54]; Hartgerink et al., 2015). As we understand RSF's model, situational cues in Cyberball would be the number of ball tosses to the participant, whereas social exclusion would be a situational characteristic. An informative design would be to systematically vary the number of ball tosses along a gradient so there are multiple levels of the IV. This would facilitate testing of non-linear functions. Moreover, outside observers could be used to code the game to estimate ex situ evaluations of exclusion. Collectively, such experimental work could prove invaluable for testing specific hypotheses about the situational factors captured by Cyberball and for producing a psychologically rich understanding of social exclusion.

To be clear, we agree with RSF that people often influence situations to create matches with their personalities. Nonetheless, such matching processes are constrained by factors including age, culture, socioeconomic status, and probably random luck. People $d o$ find themselves in situations orthogonal to their personalities on a regular basis, making such situations important to study. Bystander intervention scenarios are good example as this is an event that can happen to real people. The situational and dispositional elements that motivate behavior in these situations 
are important for both scientific and practical reasons. More importantly, many of these situational elements are amenable to manipulation as shown in classic studies.

Indeed, re-evaluating classic experimental paradigms through the lens the RSF perspective could help to validate a working taxonomy of situational characteristics and prove to be extraordinarily integrative for social psychological and personality research. These classic experimental situations might serve as low hanging fruit. Thus, in the interest of integration, we suggest that researchers complement studies of "natural" (i.e., self-selected) situations with studies of compelling "artificial" situations to further the science of situations. Accordingly, we suggest: $A$ working taxonomy of situational characteristics is particularly useful when it also allows for a systematic manipulation of cues to test causal hypotheses.

\section{Conclusion}

We had been pessimistic about the possibility of an integrated science of behavior that balances a nuanced understanding of human individuality with an equally nuanced understanding of situational attributes. The personality side of the equation has had a head start on the situation side. However, work on situational taxonomies is making significant strides thanks to RSF's work. Thus, we are excited for the future, and we believe that many valuable insights can be gained through an integration of classic experimental social psychology with personality psychology.

\section{References}

Buss, D. M. (1987). Selection, evocation, and manipulation. Journal of personality and social psychology, 53, 1214-1221.

Hartgerink, C. H. J., Van Beest, I., Wicherts, J. M., \& Williams, K. D. (Unpublished manuscript). Ordinal effects of ostracism: A meta-analysis of Cyberball studies. Retrieved from https://osf.io/ht25n/

Ozer, D. J., \& Benet-Martínez, V. (2006). Personality and the prediction of consequential outcomes. Annual Review of Psychology, 57, 401-421.

Rauthmann, J. F., Sherman, R. A., \& Funder, D. C. (in press). Principles of situation research: Towards a better understanding of psychological situations. European Journal of Personality Psychology.

Roberts, B. W., Kuncel, N. R., Shiner, R., Caspi, A., \& Goldberg, L. R. (2007). The power of personality: The comparative validity of personality traits, socioeconomic status, and cognitive ability for predicting important life outcomes. Perspectives on Psychological Science, 2, 313-345. 